\title{
Acute High Lateral Myocardial Infarction by ECG Finding
}

National Cancer Institute

\section{Source}

National Cancer Institute. Acute High Lateral Myocardial Infarction by ECG Finding. NCI

Thesaurus. Code C102593.

An electrocardiographic finding of patholog ic Q waves with accompanying ST elevation in leads I and aVL, which is suggestive of acute myocardial infarction of the high lateral wall of the left ventricle. (CDISC) 